BROOKHAVEN NATIONAL LABORATORY

Associated Universitles, Inc.

Upton, New York

ACCELERATOR DEPARTMENT

Informal Report

ISABELLE FOR VERY LOW ENERGY PP EXPERIMENTS: WHIPLASH

T. Kalogeropoulos

November 12,1971

\title{
NOTICE
}

This report was prepared as an account of work sponsored by the United States Government. Neither the United States nor the United States Atomic Energy Commission, nor any of their employees, nor sny of their contractors, subcontractors, or thefr employees, makes any warranty, express or implied, ot assumes any legal 1 lability or responsibility for the accuracy, completeness or usefulness of any information, apparatus, product or process disclosed, or represents that its use would not infringe privately owned rights. 
BNL 16813

Accelerator Department

CRISP 71-46

BROOKILAVEN NATIONAL LABORATORY

ISABELLE PROJECT

Associated Universities, Inc.

Upton, New York

ISABELLE for Very Low Energy pp Experiments: WHIPLASH

T. Kalogeropoulos*

November 12,1971 
ISABELLE for very Low Energy pp Experiments: WHIPLASH

T. Kalogeropoulos

November 12,1971

Recent observations on $N \bar{N}$ annihilations at low energies ( $15 \mathrm{MeV}$ center of mass energy) suggest that interesting physics problems lie in this energy region. The observation of $\bar{p} p \rightarrow 2 \pi^{\circ}$ at rest by Columbia-Syracuse (submitted to PRL) has seriously contradicted the decade long belief that captures at rest proceed viil S-states. Alf y'en in Physics Today V. 24(71) points out that :he annihilation cross-section at $\sim 20 \mathrm{~V}$ is crucial for the matterantimatter models of the universe. In spite of a decade of experimentation with antiprotons at low energies, we only have results on annihilation at rest (protonium in liquid hydrogen) and we know reliably the cross-sections $\geq 250 \mathrm{MeV} / \mathrm{c}$ (15 MeV cm energy).

I have considered over the years various schemes in order to be able to study low energies. Even with the most optimistic extrapolation of hardware and imagination the results came out to be discouraging. All these schemes had one point in common: the target being stationary in the laboratory system.

Starting with antiproton beams, the principal constraincs are: (a) The number of antiprotons annihilating in flight over those stopping decreases rapidly with decreasing energy. (b) The identification problem. This problem can in principle be solved by using a gas target, however, this will be done at the great expense of flux since $\delta p / p$ has to become small. Both these problems will be absent in the event that $\bar{n}$ beams could be made. I have examined 
CRISP $71-46$

the possibilities of $\bar{n}$ "beans" produced via $\bar{p} p+\ddot{n}$. The smallness of $\mathrm{d} \sigma / \mathrm{d} \Omega$ ) $180^{\circ}$ (which produces low energy $\bar{n}$ ); the difficulties of geometry and the difficulties associated with tagging the $\vec{n}$ energy by measuring the $\vec{p}_{n}$ are too great to make possible the study of $N \bar{N}$ at low energies with a stationary target.

The purpose of this note is to bring to attention that if antiproton-proton beams wil1 bestored running in the same direction (WHIPLASH mode) very small cm po energies can be achieved in contrast to the standard case (HEAD-ON) which yields very high energies. I will not discuss, here, the questions of physics in NN at low energies (eV-MeV range) except to point out that: (a) At these low energies, the Coulomb, nuclear (pion range) and annihilation potentials will be contributing all in an important way and their superposition is bound to exhibit interesting variations. (b) Since the annihilations at rest have not provided a "nice" system - via s-captures -, we may still be able to define an energy in which S-waves are dominant and those test the prediction of symmetries.

I urge that serious thought be given for storing antiprotons in ISABELLE for high energy as well as low pip experiments. The high energy ppp experiments together with the corresponding pp will give the only direct test of Pomeranchuk theorem at the highest possible energies. On the other hand, the low energy experiments will be the lowest possible energy experiments that we will ever do. It is my belief that many revealing surprises will lie at these low energies. The order of magnitude estimate for storing $\dot{p}$ without cooling made. by $R$. Palmer (BNL16013) 
CRISP 71-46

are more than adequate for doing even "exotic" p̄p experiments at low energies. This proposal gives the basic parameters which have to be considered for WHIPLASH. It is clear from the above that such a facility will be unique.

1. The Principle of WHIPLASH

The total cms energy $(E \equiv Q+2 m)$ is given by

$$
E^{2}=\left(\omega+\omega^{\prime}\right)^{2}-\left(\vec{p}+\vec{p}^{\prime}\right)^{2}
$$

where $\vec{p}, \vec{p}$ ' are the two beam momenta such that

$$
|\vec{p}-\vec{p} \cdot| \ll|\vec{p}| \text {. }
$$

With the approximation

$$
\left[1+\left\{|\vec{p}-\vec{p} \cdot|^{2}-2 \vec{p} \cdot\left(\vec{p}-\vec{p}^{\prime}\right)\right\} / \omega^{2}\right]^{1 / 2} \simeq 1+\left\{|\vec{p}-\vec{p} \cdot|^{2}-2 \vec{p} \cdot(\vec{p}-\vec{p} \cdot)\right\} \mid 2 \omega^{2}
$$

and $E+2 m \simeq 4 m$ we obtain

$$
4 m Q=\left(\vec{p}_{1}-\vec{p}_{2}^{\prime}\right)^{2}+\left(p_{n}-p_{n}^{\prime}\right)^{2}
$$

where the average $\bar{p}$ direction defines the cransverse and longitudinal components. From (1) we see that by suitable choice of the two beam momenta any $Q$ is possible. It also shows that the spreads in the longitudinal and transverse components in the beams will define the resolution in $Q(\delta Q)$, which defines the energy at which an experiment can be performed. In principle there is no limit in controlling $\delta \vec{p}_{2}$ and $\delta p_{n}$ but this will have as a consequence, corresponding reflections on the beam fluxes. Any arbitrary $\delta Q$ can be reached if the flux sacrifices are tolerable. 
CRISP $71-46$

\section{Interaction Rate}

In head-on collisions, the bunches cross each other completely while transversing the interaction region $\left(L_{i}\right)$ and consequently any oneparticle of one bunch has a chance of interacting with all other particles of the other bunch. In WHIPLASH, each particle sees only a fraction of the other bunch over the interaction region and this depends on the relative velocity (v) and the time $(\tau)$ that it takes to transverse the interaction region. This overlap factor $\left(f_{0}\right)$ is

$$
f_{0}=\frac{v \tau}{L_{b}}=\frac{v}{c} \frac{L_{i}}{L_{b}}=2 \sqrt{\frac{Q}{m}} \frac{L_{i}}{L_{b}} \leq 1
$$

where $L_{b}$ is the bunch length (assuming the same for both beams). The rates for WHIPLASH $\left(R_{w}\right)$ and head-on $\left(R_{H}\right)$, for otherwise identical beams, will be related through the overlap factor and their respective cross-sections..

$$
R_{W}=f_{\text {oi }} \cdot \frac{\sigma_{w}}{\sigma_{H}} \cdot R_{H}
$$

The $f_{0}$ cannot become greater. than one by definition but it may become large by suitable adjustment of $L_{i} / L_{b}$.

It is known that the annihilation cross-section is increasing as $1 / v$ at low energies, which is equivalent to $1 / \sqrt{Q}$, and it is likely at very low energies (where the Coulomb field will be important) to approach thie unitarity limit of $1 / v^{2}(1 / Q)$. Consequently, $f_{0} \sigma_{w}$ is likely to remain constant or increase with 
CRISP $71-46$

decreasing $Q$. At $Q=45 \mathrm{MeV}(300 \mathrm{MeV} / \mathrm{c} \mathrm{Lab}) \sigma \simeq 100 \mathrm{mb}$ and it is seen increasing as $1 / \sqrt{Q}$. Moreover, $\sigma_{H} \sim 40 \mathrm{mb}$ and thus

$$
f_{0} \frac{\sigma_{w}}{\sigma_{H}} \geq \frac{L_{i}}{L_{b}}
$$

Consequently, even for the case of $L_{i}=L_{b}, R_{w} \geq R_{H}$ ! We thus conclude that the "rate problem" is equivalent to that for head-on ISR's or it may easily become "easier". The actual rates, however, will be determined by the fluxes which can be stored compatible with the resolution required.

\section{Antiprcton Flux}

R. Palmer (BNL 16013) has estimated that in about two weeks the ISABELLE storage ring phase space can be filled with $2 \times 10^{10}$ antiprotons with $\delta \mathrm{p} / \mathrm{p}=10^{-2}$ at $2 \mathrm{GeV}$ and a half beam angular spread of $2 \mathrm{mrad}$ corresponding to a transverse momentum of $4 \mathrm{MeV} / \mathrm{c}$. In this estimate, no cooling has been assumed. The largest annihilation experiments done up to now at low energies (at rest) consist of $\sim 10^{7}$ annihilation. Clearly, the total number of $\bar{p}^{\prime} s$ within Palmer's estimate will not be a problem for good annihilation-experiments. The resolucion in $Q$ will be due to $\delta p / p$. From (1) by substituting $\delta\left(p_{n}-p_{\prime \prime}^{\prime}\right)^{2} \cong\left(p \cdot \frac{\delta p}{p}\right)^{2}$ we get

$$
\delta Q \sim \frac{1}{4 m}\left(p \cdot \frac{\delta p}{p}\right)^{2}=0.1 \mathrm{MeV} \text { । }
$$

and thus experimeits down to $100 \mathrm{KV}$ can be done with high fluxes. By decreasing $\frac{\delta p}{p}$, the resolution is increasing $\alpha\left(\frac{\delta p}{p}\right)^{2}$ while the $\bar{p}$ flux goes linearly with $\delta p / p$ and consequently a wide range of 
CRISP 71.46

Q's can be explored in the $\mathrm{KV}$ and MeV region and possibly in the ev.

Palmer also estimates that with $2 \times 10^{10} \bar{p}$ stored in the head-on configuration $10^{4}$ interaction/sec will occur. In $\$ 2$ we concluded that $R_{w} \sim R_{H}$ and consequently with $10^{10} \bar{p}$ stored many (100!) experiments, each consisting of $\sim 10^{7}$ annihilations, zan be done over the course of a fraction of a year!

\section{Experimenta1 "Kinematics"}

In contrast to the head-on experiments which have "forwardback" cones and arbitrarily large momentum transfers are possible, in WIIPLASH only a forward cone with a maximum half angle of $\sim \mathrm{m} / \mathrm{p}$ will only be present. Consequently, the $4 \pi$ geometry required for studying annihilations at low energies.with stationary targets is reduced to a small cone whose angle is controlled by the beam momentum. This, considerably simplifies the experimental requirements and it will make possible the design of experiments which are hard to do otherwise at low energies. The high energies of the annihilation products as well as the absence of a target will reduce correctionsdue to secondary interactions, Goulomb scattering etc.

\section{Protonium Beams?}

At very low energies protonium will be formed and it will be moving in free space with a momentur twice the beam momentum. In this case there will be no Stark mixing effect and the radiative transitions will compcte with annihilations. Various esti- 
CRISP 71-46

mates indicate that the radiative transitions will compete with the annihilation at the rate of $\sim 10^{10} \mathrm{sec}^{-1}$. Consequently, the protunium will transverse a distance of

$$
10^{10} \mathrm{c} \cdot \gamma=3 \times \frac{\mathrm{p}}{\mathrm{n}} \cong 6 \text { meters }
$$

at $200 \mathrm{~B}$ eV beam momentum! The radiative transition $\mathrm{X}$-rays will have higher energies on the average by the factor $\gamma$ and they will be mainly emitted forward and thus correlation experiments of annihilations from specific atomic states might be possible. 